\title{
GMR
}

\section{Association between the 8q24 rs6983267 T/G polymorphism and prostate cancer risk: a meta-analysis}

\author{
H.S. Zhu ${ }^{1 *}$, J.F. Zhang ${ }^{1 *}$, J.D. Zhou ${ }^{2}$, M.J. Zhang ${ }^{1}$ and H.X. Hu ${ }^{1}$ \\ ${ }^{1}$ Clinical Laboratory, East Suzhou Municipal Hospital, Nanjing Medical University, \\ Gusu District, Suzhou, Jiangsu Province, China \\ ${ }^{2}$ Department of Radiotherapy, East Suzhou Municipal Hospital, \\ Nanjing Medical University, Gusu District, Suzhou, Jiangsu Province, China \\ *These authors contributed equally to this study. \\ Corresponding author: H.S. Zhu \\ E-mail: xintaozhangxintao@163.com
}

Genet. Mol. Res. 14 (4): 19329-19341 (2015)

Received August 17, 2015

Accepted October 2, 2015

Published December 29, 2015

DOI http://dx.doi.org/10.4238/2015.December.29.43

\begin{abstract}
Recent studies have indicated that single nucleotide polymorphisms (SNPs) within the 8q24 region may be a risk factor for prostate cancer $(\mathrm{PCa})$. Here, we performed a meta-analysis to evaluate the association between the 8q24 rs6983267 T/G polymorphism and PCa risk. A systematic literature search was carried out in multiple electronic databases independently by two investigators. Pooled odds ratios (ORs) and $95 \%$ confidence intervals for $8 \mathrm{q} 24$ rs6983267 T/G and PCa were calculated using a fixed-effect model (the Mantel-Haenszel method). In total, 24 case-control studies from 19 articles were included in our meta-analysis. Our analysis indicated that there is a significant $\mathrm{PCa}$ risk associated with the rs6983267 polymorphism in a dominant model (GG vs GT+TT, pooled $\mathrm{OR}=1.298, \mathrm{P}<0.001)$; recessive model $(\mathrm{GG}+\mathrm{GT}$ vs $\mathrm{TT}$, pooled $\mathrm{OR}=$ 1.302, $P<0.001$ ); and homozygote comparison (GG vs TT, pooled OR $=1.494, P<0.001$ ). Similarly, in a subgroup analysis of European and Asian descent, our results revealed that there are associations between
\end{abstract}


rs6983267 T/G polymorphism and PCa susceptibility with the dominant model (GG vs GT+TT), recessive model (GG+GT vs TT), and homozygote comparison (GG vs TT). To investigate the association between rs6983267 and risk of PCa under different clinical conditions, further analyses were conducted regarding different clinical characteristics including the Gleason score, tumor stage, and PSA level to provide a more comprehensive view of PCa risk and this SNP. Publication bias was assed using the Begg test and the Egger test, and none was detected.

Key words: Prostate cancer; Meta-analysis; Polymorphism

\section{INTRODUCTION}

Prostate cancer $(\mathrm{PCa})$ is the most common cancer and third leading cause of cancerrelated deaths among males in developed countries (Crawford, 2003). Approximately 233,000 new cases were expected to be diagnosed with 29,480 estimated deaths in the USA in 2014 (Siegel et al., 2014). Despite this, the underlying etiology of PCa is poorly understood. The most recognized factors associated with PCa risk include age, ethnicity, cigarette smoking, and alcohol consumption. Additionally, it has been suggested that several genetic polymorphisms could influence susceptibility to PCa (Wang et al., 2010; Shao et al., 2012).

Identifying genetic variants that increase the risk of disease development and progression is important for improving screening regimens, diagnosis, and treatment of PCa (Cheng et al., 2008). A region on chromosome $8 q 24$ was first shown to confer PCa risk in a genome-wide linkage scan of 871 Icelandic men (Amundadottir et al., 2006). Subsequently, multiple independent studies have provided compelling evidence that has demonstrated that the risk of PCa is influenced by genetic variations in the 8 q24 region (Haiman et al., 2007; Cheng et al., 2008). One promoter polymorphism rs6983267 T/G in the 8q24 region has been reported in genome-wide association studies (GWAS) to be significantly associated with PCa risk (Yeager et al., 2007; Zheng et al., 2007). While there have been numerous studies that have investigated associations between this polymorphism and the risk of $\mathrm{PCa}$, the results have been inconsistent and inconclusive.

For these reasons, we performed a meta-analysis to offer a more comprehensive estimation of the association between rs6983267 and PCa susceptibility in two groups: Asian descent and European descent. Other than using the odds ratio (OR) derived from a corresponding pooling model, we evaluated the association between rs6983267 and PCa risk for cases with different clinical characteristics including the Gleason score, tumor stage, and prostate specific antigen (PSA) level.

\section{MATERIAL AND METHODS}

\section{Literature search and data extraction}

We searched for related articles in six databases including PubMed, ScienceDirect, Karger, Web of Science, Wiley Online Library, and Springer. To avoid omitting related literature, "8q24" or "rs6983267" and "prostate cancer" were set as key words. The search coverage included those articles published in English from 2006 to 2014. Books and other literature not related to case-control studies or not aimed at prostate cancer research were excluded. Then, full texts of the 
remaining articles were carefully examined and articles that did not contain quantified information on the genotypes of rs6983267 were removed. Finally, we checked for studies with overlapping study populations, and in those cases, we chose the more appropriate studies, which were restricted to studies with subjects of Asian and European descent.

All data were extracted independently by two reviewers (H.S. Zhu and J.F. Zhang). Preliminary evaluation was conducted based on the title and abstract, and full texts of potentially relevant studies were obtained and re-evaluated for inclusion. The following characteristics were collected from each study: year of publication, first author, race or ethnicity of samples, exact quantity of each genotype for cases and controls, Hardy-Weinberg equilibrium (HWE), P value, and genotyping method. In addition, clinical characteristic of cases such as Gleason score, tumor stage, and PSA level were collected, especially for studies with detailed genotype information. This article does not contain any studies with human participants performed by any of the authors

\section{Statistical analysis}

The statistical analyses were conducted with STATA 12 (Stata Corp LP, College Station, TX, United States). For all analyses, $P$ values less than 0.05 were considered statistically significant. HWE in cases or controls was evaluated by the chi-square test, and $\mathrm{P}$ values over 0.05 were considered as significant equilibriums. HWE was also taken as a data-extraction factor and those studies with HWE $P$ values over 0.05 were chosen for further analysis.

For more thorough analyses, three genetic models of inheritance were adopted: a dominant model (GG+GT vs TT), recessive model (GG vs GT+TT), and homozygote model (GG vs TT). In the dominant model, we investigated the distribution of genotypes GG and GT compared to genotype TT; as for the recessive model, the distribution of genotype GG compared to genotypes GT and TT was analyzed; in the homozygote model, we used TT as reference genotype and investigated the distribution of GG.

For each study, the quantities of the three genotypes in cases and controls were used as pooled data. As for the pooling analyses, a Mantel-Haenszel (M-H) fixed-effect model was applied to analyze datasets with insignificant heterogeneity and a DerSimonian and Laird (D-L) randomeffects model was used for datasets with clear heterogeneity. In our analyses, the heterogeneity among studies was evaluated using the $\mathrm{I}^{2}$ index. The higher the $\mathrm{I}^{2}$ statistic, the more significant the heterogeneity was considered. Specifically, when $I^{2}$ was less than $50 \%$, we considered there to be no significant heterogeneity among the pooled data, and then the $\mathrm{M}-\mathrm{H}$ model was applied; for $\mathrm{I}^{2}$ greater than $75 \%$, heterogeneity was considered to exist and the D-L model was adopted; otherwise, both models were applied. For each analysis, the $\mathrm{M}-\mathrm{H}$ model was used first to test for heterogeneity, and then an adequate model was chosen based on the test result of $\mathrm{I}^{2}$ statistic. The pooled odds ratio (OR) and 95\% confidence interval were calculated with the corresponding model, and the associated forest plots were generated to summarize the results.

As for the expanded evaluation, factors including the Gleason score, tumor stage, and PSA level were chosen, and their corresponding genotypes were collected, and risk allelic ORs comparing cases with controls were calculated to see whether there were differences between the groups with regards to the distinct clinical characteristics.

To evaluate publication bias, Begg funnel plots were generated on the basis of the analysis result and database size. The more asymmetric the funnel plot appeared, the more publication bias was potentially present. Meanwhile, Egger test was also performed for further bias investigation. For the Egger test, the significance level was set as $P<0.05$. 


\section{RESULTS}

\section{Characteristics of the studies included}

In total, 187 papers were retrieved after the first search, among which 78 articles contained case-control studies targeting prostate cancer. After further evaluation, we found only 24 casecontrol studies from 19 articles that were suitable for our meta-analysis of rs6983267 (Haiman et al., 2007; Yeager et al., 2007; Zheng et al., 2007; Cheng et al., 2008; Salinas et al., 2008; Terada et al., 2008; Wokolorczyk et al., 2008; Beuten et al., 2009; Liu et al., 2009; Penney et al., 2009; Zheng et al., 2009; Liu et al., 2011; Papanikolopoulou et al., 2011; Joung et al., 2012; Brankovic et al., 2013; Ho et al., 2013; Chan et al., 2013; Zhao et al., 2013; Oskina et al., 2014). Among these, 16 studies included populations of European descent and 8 included populations of Asian descent. The flow chart of study inclusion and reasons for exclusion are presented in Figure 1. Characteristics of the studies included in the meta-analysis are presented in Table 1. All data in these studies were related to the association between 8q24 rs6983267 T/G polymorphism and human PCa susceptibility.

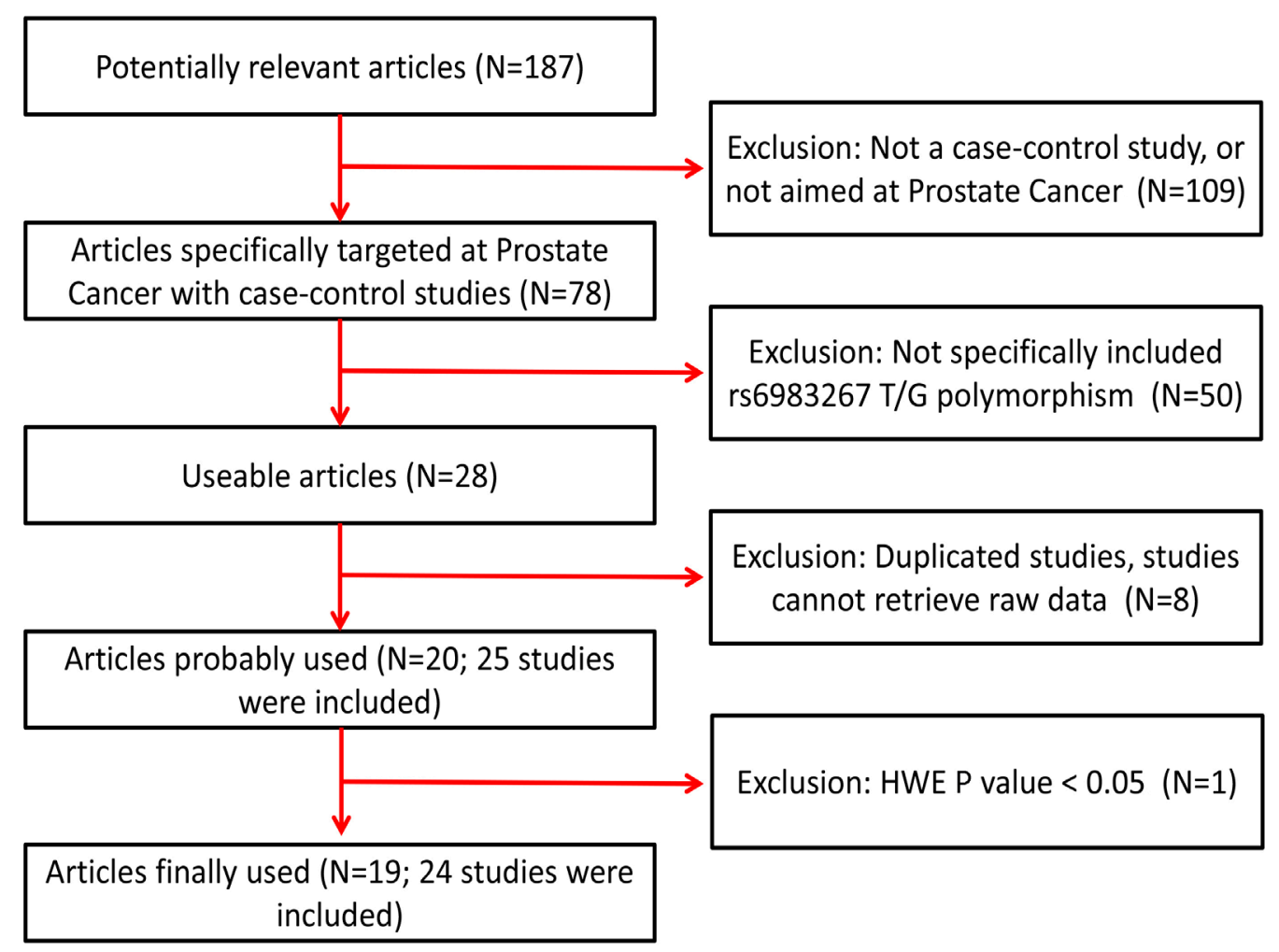

Figure 1. Flow chart of screened, excluded, and included studies with specific reasons for exclusion from the metaanalysis. 
Polymorphism association with prostate cancer

19333

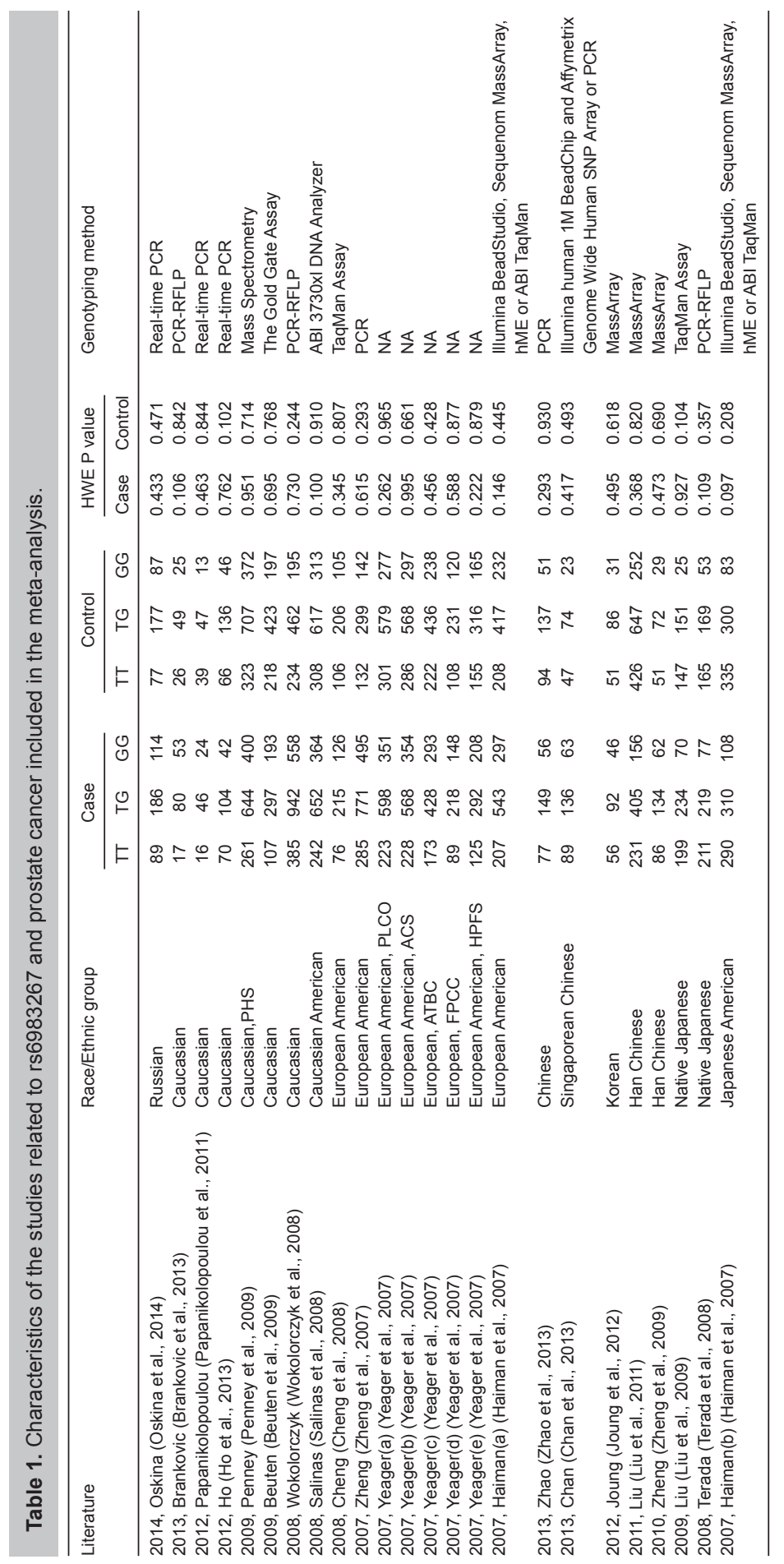

Genetics and Molecular Research 14 (4): 19329-19341 (2015) 


\section{Evaluation of the association between the rs6983267 polymorphism and prostate cancer}

There were 24 case-control studies included in our meta-analyses for the association between polymorphism rs6983267 and PCa. For the included studies, low heterogeneity was observed under all three models (for the dominant model, $\mathrm{I}^{2}=30.2 \%, \mathrm{P}=0.082$; for the recessive model, $\mathrm{I}^{2}=2.7 \%, \mathrm{P}=0.424$; and for the homozygote model, $\left.\mathrm{I}^{2}=31.2 \%, \mathrm{P}=0.074\right)$. Based on these results, the $\mathrm{M}-\mathrm{H}$ model was applied using the three different genetic models (recessive, dominant, and homozygote). Detailed results of the meta-analysis are shown in Table 2. Overall, when all eligible studies were pooled into the meta-analyses, we found that a significant PCa risk was associated with the rs6983267 polymorphism in the dominant model (GG vs GT+TT, pooled OR $=1.298,95 \% \mathrm{Cl}=1.232-1.368, \mathrm{P}<0.001)$; recessive model $(\mathrm{GG}+\mathrm{GT}$ vs TT, pooled OR $=1.302$, $95 \% \mathrm{Cl}=1.236-1.372, \mathrm{P}<0.001)$; and homozygote model $(\mathrm{GG}$ vs TT, pooled OR $=1.494,95 \% \mathrm{Cl}$ $=1.400-1.595, \mathrm{P}<0.001)$. The forest plots of the three models are shown in Figure 2.

Table 2. Meta-analysis of rs6983267 with the dominant model (GG+GT vs TT), recessive model (GG vs GT+TT), and homozygote model (GG vs TT) for the entire database, populations with European descent, and populations with Asian descent.

\begin{tabular}{|c|c|c|c|c|c|c|c|c|c|}
\hline \multirow[t]{2}{*}{ Analysis model } & \multirow[t]{2}{*}{ Pooling model } & \multicolumn{2}{|c|}{ Heterogeneity } & \multicolumn{4}{|c|}{ OR $(95 \% \mathrm{Cl})$} & \multicolumn{2}{|c|}{ Publication bias } \\
\hline & & $I^{2}(\%)$ & $P$ value & Overall & Lower & Upper & $P$ value & $\begin{array}{l}\text { Begg test } \\
\text { ( } P \text { value) }\end{array}$ & $\begin{array}{c}\text { Egger test } \\
(\mathrm{P} \text { value })\end{array}$ \\
\hline \multicolumn{10}{|c|}{ For entire database } \\
\hline Dominant & $\mathrm{M}-\mathrm{H}$ & 30.2 & 0.082 & 1.298 & 1.232 & 1.368 & $<0.001$ & 0.47 & 0.854 \\
\hline Recessive & $\mathrm{M}-\mathrm{H}$ & 2.7 & 0.424 & 1.302 & 1.236 & 1.372 & $<0.001$ & 1.17 & 0.255 \\
\hline Homozygote & $\mathrm{M}-\mathrm{H}$ & 31.2 & 0.074 & 1.494 & 1.400 & 1.595 & $<0.001$ & 0.37 & 0.498 \\
\hline \multicolumn{10}{|c|}{ For populations with European descent } \\
\hline Dominant & $\mathrm{M}-\mathrm{H}$ & 41.8 & 0.040 & 1.346 & 1.266 & 1.432 & $<0.001$ & 0.05 & 0.469 \\
\hline Recessive & $\mathrm{M}-\mathrm{H}$ & 4.0 & 0.407 & 1.317 & 1.244 & 1.395 & $<0.001$ & 1.13 & 0.229 \\
\hline Homozygote & $\mathrm{M}-\mathrm{H}$ & 38.8 & 0.057 & 1.541 & 1.432 & 1.658 & $<0.001$ & 0.86 & 0.289 \\
\hline \multicolumn{10}{|c|}{ For populations with Asian descent } \\
\hline Dominant & $\mathrm{M}-\mathrm{H}$ & 0 & 0.940 & 1.179 & 1.067 & 1.303 & 0.001 & 0.87 & 0.680 \\
\hline Recessive & $\mathrm{M}-\mathrm{H}$ & 0 & 0.431 & 1.228 & 1.080 & 1.397 & 0.002 & 1.36 & 0.094 \\
\hline Homozygote & $\mathrm{M}-\mathrm{H}$ & 0 & 0.594 & 1.328 & 1.149 & 1.533 & $<0.001$ & 1.11 & 0.267 \\
\hline
\end{tabular}

Similarly, we performed subgroup analyses as shown in Table 2. For the subgroup analyses, the $\mathrm{M}-\mathrm{H}$ fixed-effect model was first applied on each subgroup dataset using the three genetic models (recessive, dominant, and homozygote) to test the heterogeneity. For the 16 studies with populations of European descent, the $\mathrm{M}-\mathrm{H}$ model was applied for all three genetic models, which revealed low heterogeneity (for the dominant model, $\mathrm{I}^{2}=41.8 \%, \mathrm{P}=0.040$; for the recessive model, $\mathrm{I}^{2}=4.0 \%, \mathrm{P}=0.407$; and for the homozygote model, $\mathrm{I}^{2}=38.8 \%, \mathrm{P}=0.057$ ). As shown in Table 2 , significant $\mathrm{PCa}$ risks associated with the rs6983267 polymorphism were found using the recessive model $(\mathrm{GG}+\mathrm{GT}$ vs TT, pooled OR $=1.317,95 \% \mathrm{Cl}=1.244-1.395, \mathrm{P}<0.001)$; the dominant model ( $\mathrm{GG}$ vs GT+TT, pooled OR $=1.346,95 \% \mathrm{Cl}=1.266-1.432, \mathrm{P}<0.001$ ); and the homozygote model ( $G G$ vs TT, pooled $O R=1.541,95 \% \mathrm{Cl}=1.432-1.658, P<0.001)$. The corresponding forest plots for the three genetic models are shown in Figure 3. For the 8 studies with populations of Asian descent, the $\mathrm{M}-\mathrm{H}$ model was also applied for all three genetic models and low heterogeneity was found (for the dominant model, $\mathrm{I}^{2}=0, \mathrm{P}=0.940$; for the recessive model, $\mathrm{I}^{2}=0, \mathrm{P}=0.431$; and for the homozygote model, $\left.\mathrm{I}^{2}=0, \mathrm{P}=0.594\right)$. Additionally, significant $\mathrm{PCa}$ risks associated with the rs6983267 polymorphism were observed in the Asian group using the dominant model (GG vs 
GT+TT, pooled OR = 1.179, 95\%Cl = 1.067-1.303; $\mathrm{P}=0.001)$, recessive model $(\mathrm{GG}+\mathrm{GT}$ vs TT, pooled $\mathrm{OR}=1.228,95 \% \mathrm{Cl}=1.080-1.397, \mathrm{P}=0.002)$, and homozygote model (GG vs TT, pooled $\mathrm{OR}=1.328,95 \% \mathrm{Cl}=1.149-1.533, \mathrm{P}<0.001)$. The corresponding forest plots for the three genetic models are shown in Figure 4.
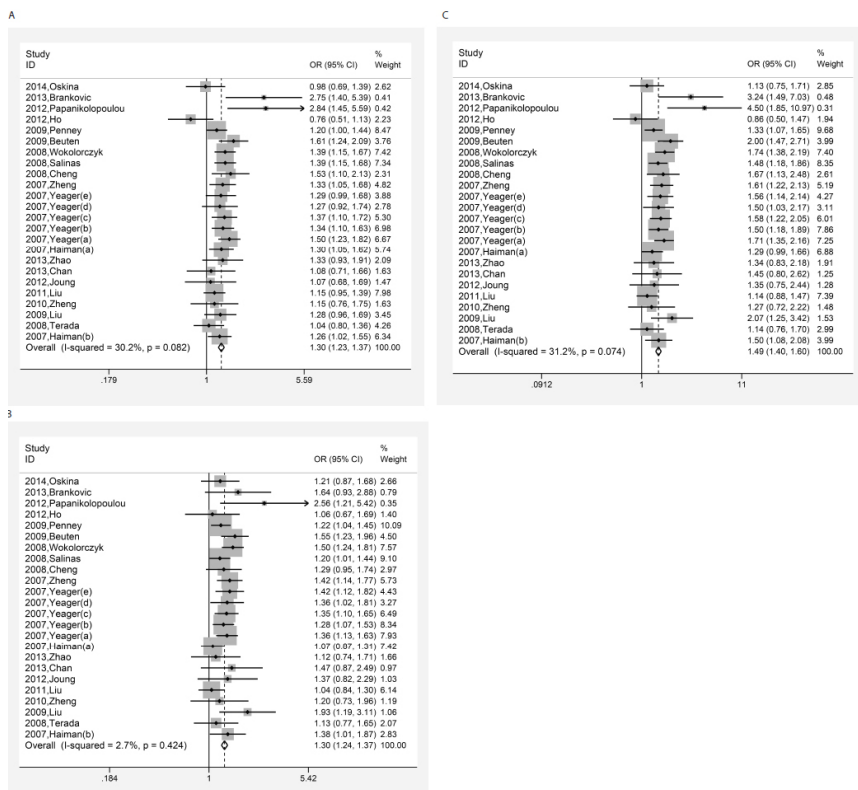

Figure 2. Forest plots of the studies included for the total populations using A. dominant model, B. recessive model, and $\mathbf{C}$. homozygote comparison.
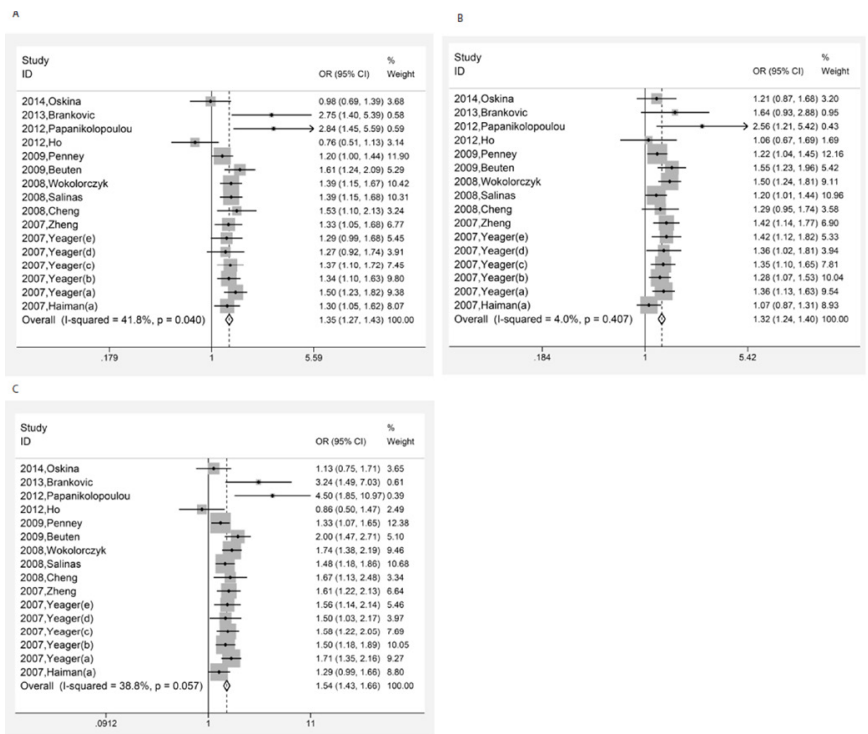

Figure 3. Forest plots of studies with populations of European descent for A. dominant model, B. recessive model, and $\mathbf{C}$. homozygote comparison. 


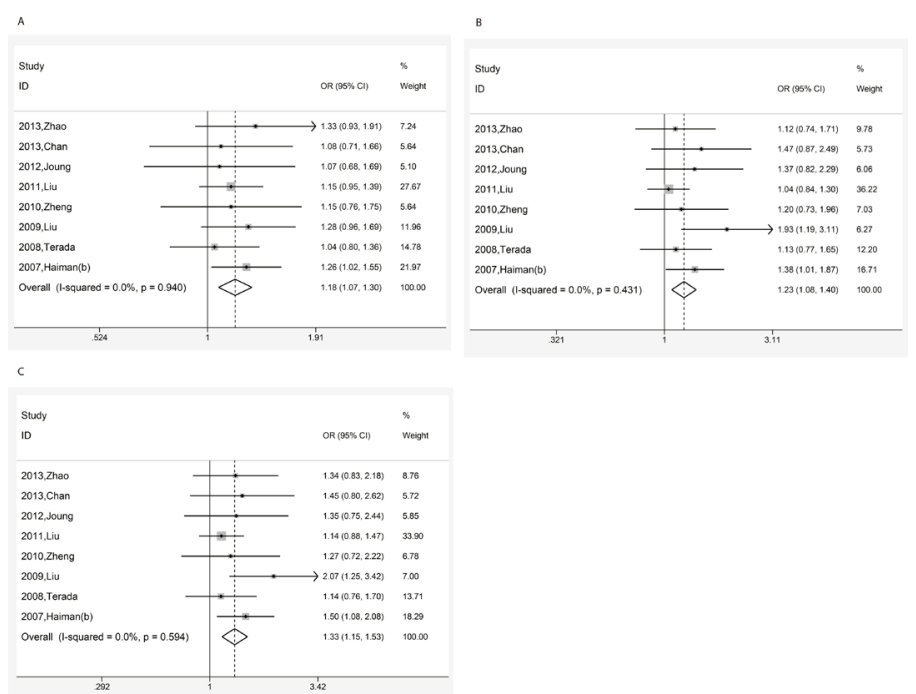

Figure 4. Forest plots of studies with populations of Asian descent for A. dominant model, B. recessive model, and C. homozygote comparison.

\section{Expanded evaluation with different clinical characteristics}

In addition to the overall and subgroup analyses, we evaluated the risk of rs6983267 with different clinical characteristics including the Gleason score, tumor stage, and PSA level, and the detailed results are shown in Table 3. As for cases with Gleason scores less than or equal to 7, the risk allelic OR was $1.195(95 \% \mathrm{Cl}=1.082-1.318, \mathrm{P}<0.001)$, and for cases with Gleason scores greater than 7 , the risk allelic OR was $1.164(95 \% \mathrm{Cl}=1.023-1.323, \mathrm{P}=0.021)$. As for cases with tumor stages between 1 and 2 , the risk allelic $\mathrm{OR}$ was $1.261(95 \% \mathrm{Cl}=1.082-1.471, \mathrm{P}=0.003)$, and for cases with tumor stages between 3 and 4 , the risk allelic OR was $1.275(95 \% \mathrm{Cl}=1.031$ $1.577, \mathrm{P}=0.025)$. As for cases with PSA levels less than or equal to $10 \mathrm{ng} / \mathrm{mL}$, the risk allelic OR was $1.236(95 \% \mathrm{Cl}=1.041-1.468 ; \mathrm{P}=0.015)$, and for cases with PSA levels greater than $10 \mathrm{ng} /$ $\mathrm{mL}$, the risk allelic OR was $1.214(95 \% \mathrm{Cl}=1.031-1.430, \mathrm{P}=0.020)$. The corresponding forest plots are shown as Figure 5.

\section{Publication bias}

To test for publication bias, both Begg and Egger tests were performed for the entire database. Results of the publication bias analysis are shown in Table 2, and no publication bias was observed under any of the three genetic models (all $P$ values for the Begg test and Egger test were greater than 0.05). Additionally, after samples were stratified by ethnicity, no publication bias was observed under any model (all $P$ values of Begg test and Egger test were greater than 0.05). Funnel plots for entire database and two subgroups are separately summarized in Figure 6 and 7. Similar evaluations were also conducted in analyses of the clinical characteristics, and no publication bias was observed except for the group of Gleason score $\leq 7$ (Egger test $P=0.005$ ). The corresponding funnel plots are shown as Figure 8. 
Polymorphism association with prostate cancer

19337

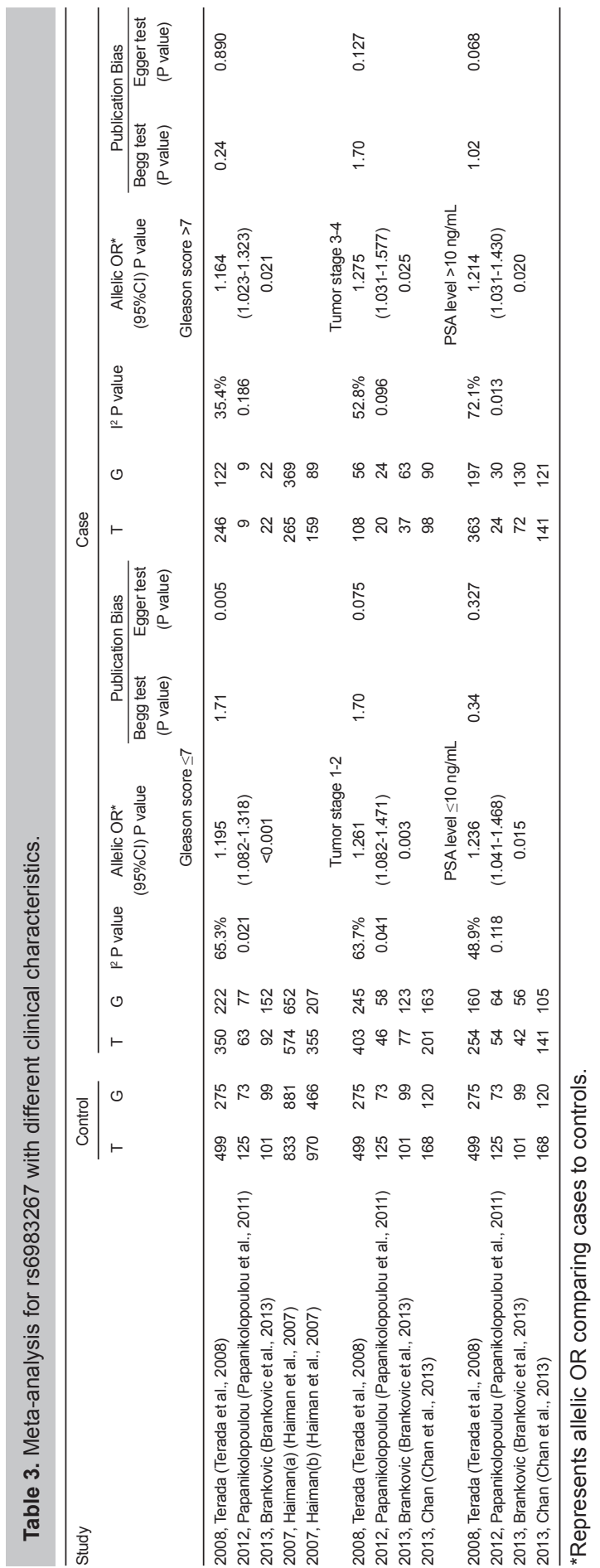



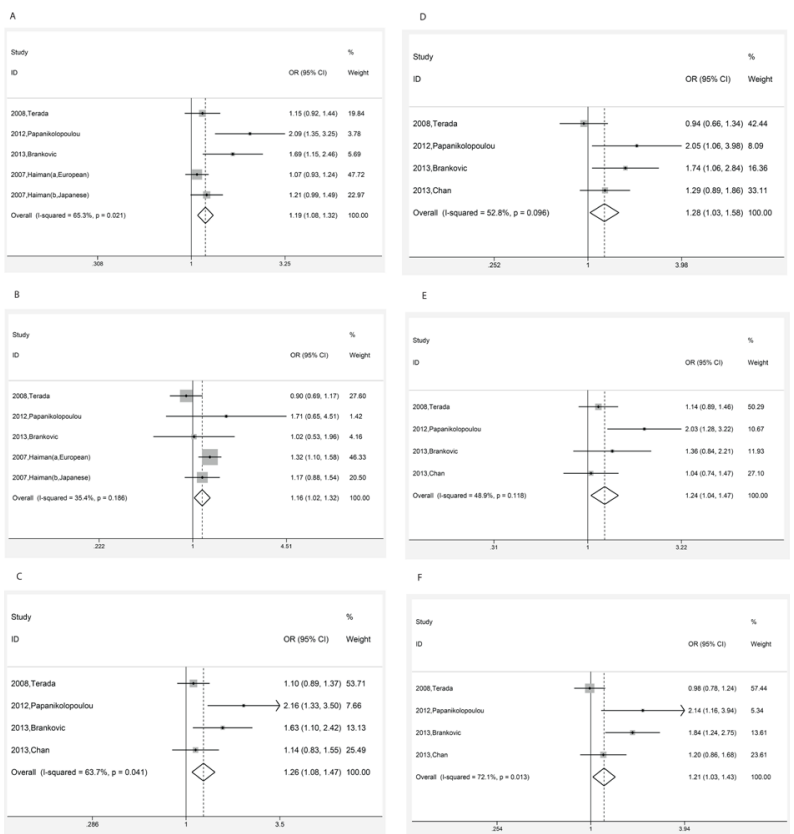

Figure 5. Forest plots of studies with different clinical characteristics for $\mathbf{A}$. cases with Gleason scores $\leq 7$, B. cases with Gleason scores >7, C. cases with tumor stage 1 to 2, D. cases with tumor stage 3 to 4 , E. cases with PSA levels $\leq 10 \mathrm{ng} / \mathrm{mL}$, and F. cases with PSA levels $>10 \mathrm{ng} / \mathrm{mL}$.
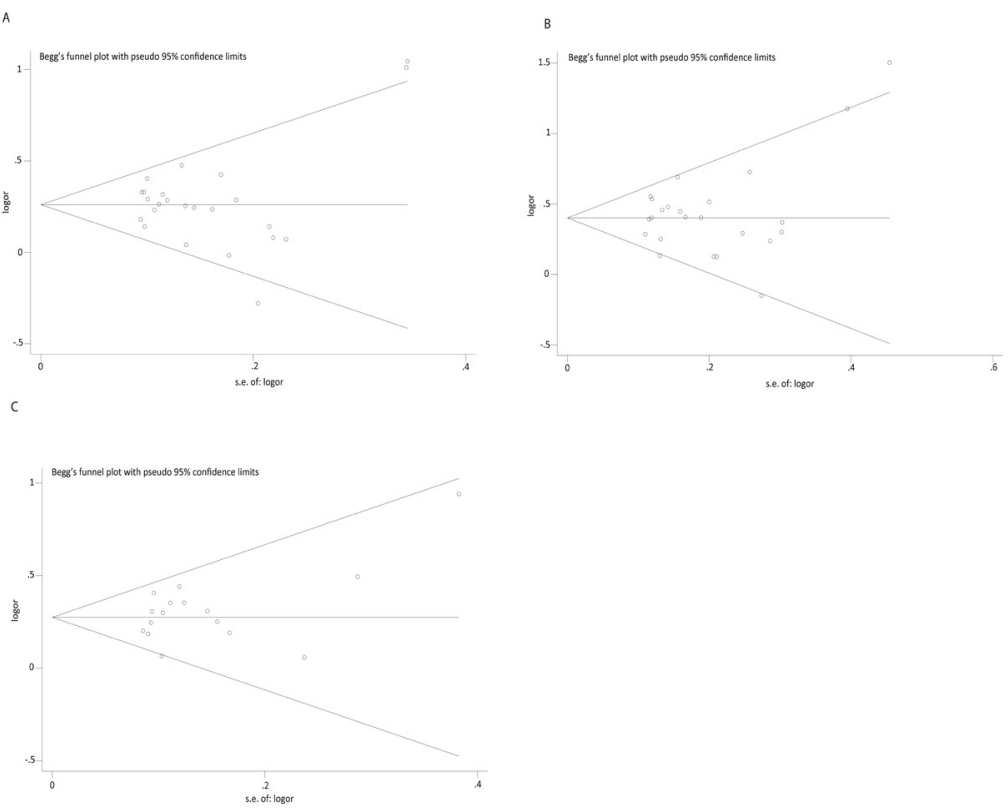

Figure 6. Funnel plots of studies with overall populations for A. dominant model, B. recessive model, and C. homozygote comparison. 


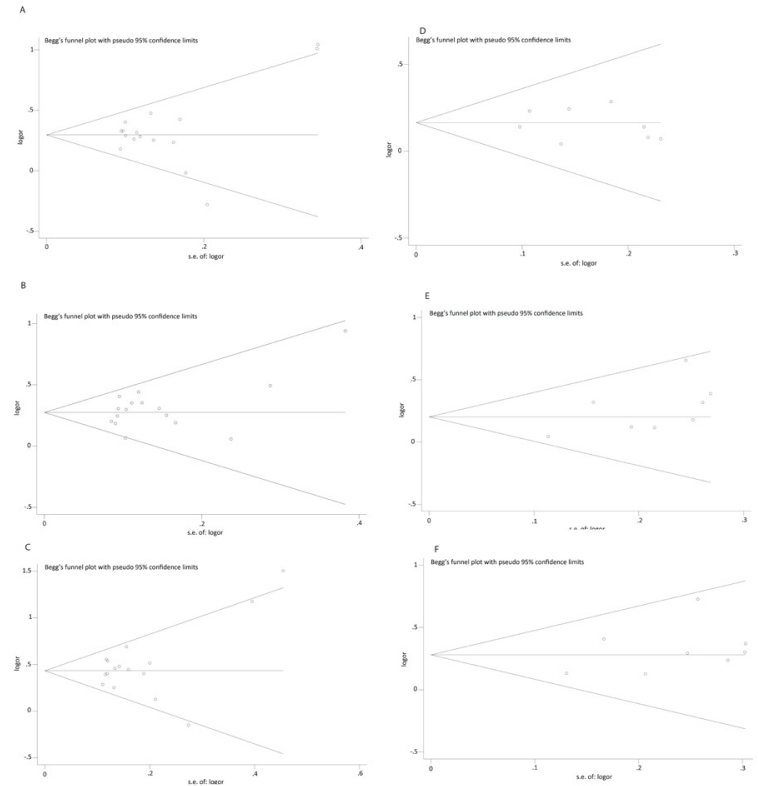

Figure 7. Funnel plots of studies with populations of European and Asian descent for $\mathbf{A}$. dominant model for European populations, B. recessive model for European populations, C. homozygote comparison for European populations, D. dominant model for Asian populations, E. recessive model for Asian populations, and F. homozygote comparison for Asian populations.

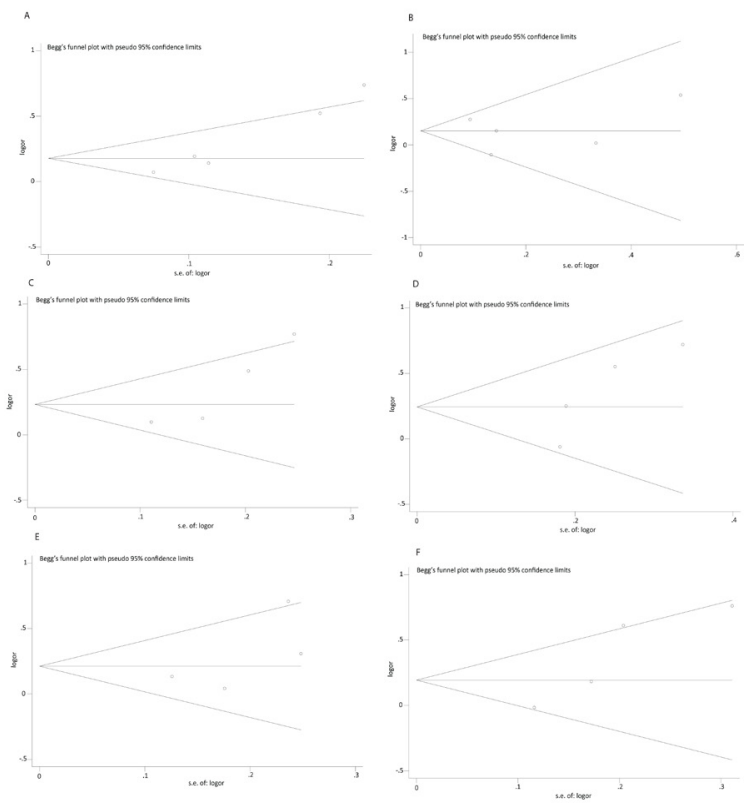

Figure 8. Funnel plots of studies with different clinical characteristics samples for A. cases with Gleason scores $\leq 7$, B. cases with Gleason scores $>7$, C. cases with tumor stage 1 to 2, D. cases with tumor stage 3 to 4 , E. cases with PSA levels $\leq 10 \mathrm{ng} / \mathrm{mL}$, and F. cases with PSA levels $>10 \mathrm{ng} / \mathrm{mL}$. 


\section{DISCUSSION}

In the current study, we analyzed the association between polymorphism rs6983267 and the risk of PCa for individuals of European and Asian descent. Although previous meta-analyses have investigated the association between rs6983267 and PCa, we performed a more detailed analysis with a larger data pool that included the most up-to-date research. Our results revealed that polymorphism rs6983267 was associated with the risk of PCa development for individuals of both European and Asian descent.

Although a prior meta-analysis investigated rs6983267 in $\mathrm{PCa}$, the study only included case and control numbers as the dataset without the specific rs6983267 genotypes. Moreover, the previous meta-analysis for rs6983267 was completed in 2012, and the data herein includes those from European and Asian populations collected from 2006 to 2014. Therefore, compared to the previous meta-analysis, two new studies for the European group and three new studies for the Asian group have been included in addition to the specific genotype data. Furthermore, the previous metaanalysis only showed that there was a higher frequency of the rs6983267 polymorphism in PCa cases than in controls in European populations, whereas no significant differences were detected in Asian populations. However, in the current study, PCa cases in both the European and Asian groups had a significant increase in the frequency of genotype GG+GT, genotype GG, and allele G.

In the present study, the $\mathrm{I}^{2}$ statistics were calculated to test for heterogeneity. For all included studies, low heterogeneity existed under the dominant, recessive, and homozygote models. However, the heterogeneity decreased when the samples were stratified according to ethnicity. For the European studies, low heterogeneity was observed under the dominant, recessive, and homozygote models, while no statistically significant heterogeneity was observed under any model in the Asian studies. Our analysis indicated that ethnicity may play an important role in genetic heterogeneity of rs6983267. Publication bias is another important factor that can affect the quality of meta-analyses. We found no significant publication bias for any model as determined by funnel plot analysis and Egger test (all P-values of Egger test were <0.05) (Table 2 and Figure 6 and 7 ).

There were several limitations of this study. First, of the 24 studies included for analysis, 8 included populations of Asian descent, but accounted for only $6.52 \%$ of the total study population. Second, because the study populations were from 10 countries and the controls were not uniform, the results should be interpreted cautiously. Third, the genetic heterogeneity among different populations emphasizes the need to interpret the results with caution.

The current meta-analysis suggests that rs6983267 T/G is associated with susceptibility to PCa in individuals of both European and Asian descent, and more specifically, the genotype $G G+G T$, genotype $G G$, and allele $G$ may significantly increase the risk of PCa in those two groups. Because of the small size of the database, larger and well-designed studies based on different subgroups are needed to confirm our results, especially for Asian populations.

\section{Conflicts of interest}

The authors declare no conflict of interest.

\section{REFERENCES}

Amundadottir LT, Sulem P, Gudmundsson J, Helgason A, et al. (2006). A common variant associated with prostate cancer in 
European and African populations. Nat. Genet. 38: 652-658.

Beuten J, Gelfond JA, Martinez-Fierro ML, Weldon KS, et al. (2009). Association of chromosome $8 q$ variants with prostate cancer risk in Caucasian and Hispanic men. Carcinogenesis 30: 1372-1379.

Brankovic AS, Brajuskovic GN, Mircetic JD, Nikolic ZZ, et al. (2013). Common variants at 8q24 are associated with prostate cancer risk in Serbian population. Pathol. Oncol. Res. 19: 559-569.

Chan JY, Li H, Singh O, Mahajan A, et al. (2013). 8q24 and 17q prostate cancer susceptibility loci in a multiethnic Asian cohort. Urol. Oncol. 31: 1553-1560.

Cheng I, Plummer SJ, Jorgenson E, Liu X, et al. (2008). 8q24 and prostate cancer: association with advanced disease and meta-analysis. Eur. J. Hum. Genet. 16: 496-505.

Crawford ED (2003). Epidemiology of prostate cancer. Urology 62: 3-12.

Haiman CA, Patterson N, Freedman ML, Myers SR, et al. (2007). Multiple regions within $8 q 24$ independently affect risk for prostate cancer. Nat. Genet. 39: 638-644.

Ho CK, Haley L, Wei J and Habib FK. (2012). Analysis of prostate cancer association with four single-nucleotide polymorphisms from genome-wide studies and serum phyto-estrogen concentrations. Prostate Cancer Prostatic Dis. 15: 365-368.

Joung JY, Park S, Yoon H, Lee SJ, et al. (2012). Association of common variations of 8q24 with the risk of prostate cancer in Koreans and a review of the Asian population. BJU Int. 110: E318-325.

Liu F, Hsing AW, Wang X, Shao Q, et al. (2011). Systematic confirmation study of reported prostate cancer risk-associated single nucleotide polymorphisms in Chinese men. Cancer Sci. 102: 1916-1920.

Liu M, Kurosaki T, Suzuki M, Enomoto Y, et al. (2009). Significance of common variants on human chromosome $8 q 24$ in relation to the risk of prostate cancer in native Japanese men. BMC Genetics 10: 37.

Oskina NA, Boyarskikh UA, Lazarev AF, Petrova VD, et al. (2014). A replication study examining association of rs6983267, rs10090154, and rs1447295 common single nucleotide polymorphisms in 8q24 region with prostate cancer in Siberians. Urol. Oncol. 32: 37.e7-12.

Papanikolopoulou A, Landt O, Ntoumas K, Bolomitis S, et al. (2011). The multi-cancer marker, rs6983267, located at region 3 of chromosome $8 \mathrm{q} 24$, is associated with prostate cancer in Greek patients but does not contribute to the aggressiveness of the disease. Clin. Chem. Lab. Med. 30: 379-385.

Penney KL, Salinas CA, Pomerantz M, Schumacher FR, et al. (2009). Evaluation of 8q24 and 17q Risk Loci and Prostate Cancer Mortality. Clin. Cancer Res. 15: 3223-3230.

Salinas CA, Kwon E, Carlson CS, Koopmeiners JS, et al. (2008). Multiple independent genetic variants in the 8q24 region are associated with prostate cancer risk. Cancer Epidemiol. Biomarkers Prev. 17: 1203-1213.

Shao N, Wang Y, Lu K, Jiang WY, et al. (2012). Role of the functional MKK4 promoter variant (-1304T>G) in a decreased risk of prostate cancer: case-control study and meta-analysis. J. Cancer Res. Clin. Oncol. 138: 1531-1539.

Siegel R, Ma J, Zou Z and Jemal A (2014). Cancer statistics, 2014. CA Cancer J. Clin. 64: 9-29.

Terada N, Tsuchiya N, Ma Z, Shimizu Y, et al. (2008). Association of genetic polymorphisms at $8 q 24$ with the risk of prostate cancer in a Japanese population. Prostate 68: 1689-1695.

Wang B, Wang D, Zhang D, Li A, et al. (2010). Pro variant of TP53 Arg72Pro contributes to esophageal squamous cell carcinoma risk: evidence from a meta-analysis. Eur. J. Cancer Prev. 19: 299-307.

Wokolorczyk D, Gliniewicz B, Sikorski A, Zlowocka E, et al. (2008). A range of cancers is associated with the rs6983267 marker on chromosome 8. Cancer Res. 68: 9982-9986.

Yeager M, Orr N, Hayes RB, Jacobs KB, et al. (2007). Genome-wide association study of prostate cancer identifies a second risk locus at 8q24. Nat. Genet. 39: 645-649.

Zhao CX, Liu M, Wang JY, Xu Y, et al. (2013). Association of 8 loci on chromosome 8q24 with prostate carcinoma risk in northern Chinese men. Asian Pac. J. Cancer Prev. 14: 6733-6738.

Zheng SL, Sun J, Cheng Y, Li G, et al. (2007). Association between two unlinked loci at 8q24 and prostate cancer risk among European Americans. J. Natl. Cancer Inst. 99: 1525-1533.

Zheng SL, Hsing AW, Sun J, Chu LW, et al. (2009). Association of 17 prostate cancer susceptibility loci with prostate cancer risk in Chinese men. Prostate. 70: 425-432. 